\title{
A Survey on Computational Intelligence Techniques used in LNA Design
}

\author{
Laura-NicoletaIvanciu \\ Bases of Electronics Department, Technical University of Cluj-Napoca, Romania
}

\begin{abstract}
Manual design of integrated circuits has lately become obsolete, given the fact that time constraints are more and more present. Hence, designers have shifted towards automatic approaches, using various software tools, which not only ensure a speedy result, but also provide a fertile environment for further designs. Many automated design processes that address integrated circuits include one or more computational intelligence techniques, such as genetic algorithms, multi-objective optimization, neural networks or fuzzy logic. The paper presents a survey on how these techniques are used in low noise amplifiers (LNA) design. The LNA is a crucial block in the structure of a communications receiver and must meet some strict specifications. A literature review of the up-to-date papers available on the subject classifies them, based on the design technique used, and comments the main categories. The paper also identifies some issues that have not been approached so far and indicates possible future directions.
\end{abstract}

Keywords: computational intelligence, genetic algorithm, low noise amplifier, optimization

\section{INTRODUCTION}

Technological advances of the past decade have shown that traditional (manual) circuit design has become obsolete. These days, automated processes are more and more used in the design loop, making the entire process fast and less faulty. However, in many cases, the intervention of a human expert is still needed, for example when the design process comes up with more than one possible solution. The final choice remains a question of human intervention. Current trends in circuit design converge towards automating the design process and obtaining a final version of the circuit, by evolving a previous one.

This paper addresses the modern methods used to design a well-known circuit, present in all communications receivers, namely theLNA (low noise amplifier). Design of an LNA using traditional techniques implies a series of calculations, in order to correctly size the components of the circuit ([1], [2]).

Computational intelligence is an umbrella concept that includes practical adaptation and selforganization concepts, and also algorithms and implementations that facilitate an intelligent behavior, in a complex and variable environment [3]. Computational intelligence is successfully applied in solving incompletely described problems or problems that are described using formal models, implying highly consuming algorithms. In comparison with artificial intelligence and hard computing, computational intelligence is able to generalize and work with imprecise data, exhibits fault and noise tolerance, and can also work in complex and variable environments ([4], [5]). Computational intelligence techniques include evolutionary computation, fuzzy logic, neural networks, self-organizing maps, etc.

Circuit synthesis using computational intelligence techniques is achieved using two approaches [6]:

- Optimization of the formal representation of the circuit's function and circuit design based on it. Generally, a genetic algorithm is used for optimization.

- Evolvable hardware (EHVW) approach - starts with randomly generated and connected circuit elements and evolves towards fulfilling the given specifications. Less common topologies can be obtained, given that the traditional design process is abandoned.

The paper is structured as follows: Section II presents fundamental LNA knowledge, including means of performance evaluation; Section III briefly describes the most common computational intelligence technique used in LNA design, namely multi-objective optimization; Section IV analyzes and compares present LNA designs using computational intelligence techniques; finally, Section V draws some conclusions and points out future development directions.

\section{LNAFUNDAMENTALS}

An LNA amplifies the signal applied at its input, and should add as little noise as possible. In a radiofrequency receiver front-end, the LNA follows the RF filter, thus needing impedance matching. A common value for the input impedance of the LNA is $50 \Omega([1],[2])$. The output signal of the LNA is then passed through a mixer or another filter, based on the architecture. Regardless of the positioning of the LNA in the receiver chain, input and output matching networks are present, as suggested in Fig. 1. The core of an LNA is a 
radiofrequency transistor, usually MOS. Its size varies with technology and influences the overall parameters of the amplifier. Additional structures can be present, for DC biasing networks, frequency compensation or increased gain [1][2].

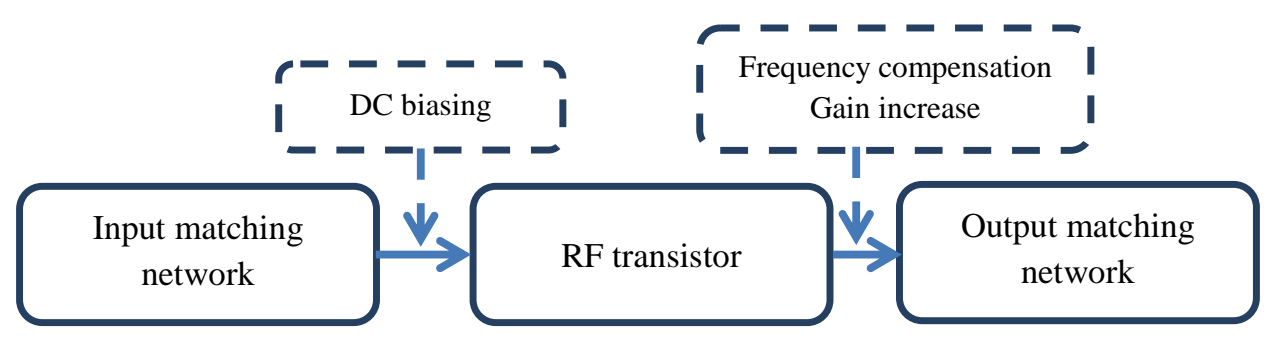

Figure 1. Schematic model of an LNA

LNA topologies can be divided into two main categories: common gate LNA, suitable for wideband applications, and inductively degenerated common source (IDCS) LNA, fitted for narrow band LNAs.

Performance evaluation of an LNA is usually achieved by means of a set of parameters, such as: gain, noise figure, nonlinearity parameters, stability, and various figures of merit.

There are six types of gain for an LNA, but only three of them are commonly used, namely: the transducer power gain, the operating power gain and the available power gain. There is no need for simulation to compute these gains, assuming one knows the values of the S-parameters.

The transducer power gain of an LNA is defined as:

The operating power gain of an LNA is:

$$
G_{T}=\left|S_{21}\right|^{2}
$$

The available power gain is computed as:

$$
G_{P}=\left|S_{21}\right|^{2} /\left(1-\left|S_{11}\right|^{2}\right)
$$

The noise figure $(\mathrm{NF})$ depends on the center frequency $\left(\mathrm{f}_{0}\right)$ of the designed LNA and some technology parameters. The computed NF can differ from the one obtained via simulation.

The second and third order intercept points (IIP2, IIP3) are located at the crossing between the fundamental component and the second or third order intermodulation product. The 1-dB compression point $(\mathrm{P} 1 \mathrm{~dB})$ is the point where the real output power growth curve is $1 \mathrm{~dB}$ under the ideal one (saturation region). Together with P1dB, IIP2 and IIP3 represent the circuit's nonlinearity parameters ([2]).

The stability factor $\mathrm{K}$ (also known as Rollett stability factor) is computed as ([1], [2]):

$$
\begin{array}{r}
K=\frac{1-\left|S_{11}\right|^{2}-\left|S_{22}\right|^{2}+|\Delta|^{2}}{2\left|S_{21} * S_{12}\right|} \\
\Delta=S_{11} * S_{22}-S_{12} * S_{21}
\end{array}
$$

For $K>1$ and $\Delta<1$, the circuit is unconditionally stable.

Different figures of merit (FOMs) can be defined, as linear combinations of the previously mentioned parameters (see (5) as an example). These FOMs come in handy when comparing different types of designs. [7]shows how FOMs can be extracted from an LNA design, using a modified GA technique.

$$
F O M=\frac{G[a b s] * I I P 3[\mathrm{~mW}]}{P_{\text {supply }}[\mathrm{mW}]} * \frac{1}{(N F-1)[a b s]} * f[G H Z]
$$

\section{MULTI-OBJECTIVE OPTIMIZATION}

Using GAs in the design process of a circuit defines two main directions: single and multi-objective optimization. While single objective optimization does not allow multiple competing objectives to be accounted for explicitly, multi-objective optimization allows different objectives to be treated separately and simultaneously during the optimization process [8]. A circuit simulator is usually introduced in the optimization loop, the most common being HSPICE, ADS or Cadence.

Genetic Algorithms (GAs) are a particular class of Evolutionary Algorithms, that use biology inspired techniques such as selection, mutation, crossover and inheritance. A general multi-objective optimization problem (MOP) can be written as follows ([9]):

Find a vector $x$ that optimizes

Subject to:

$$
f(x)=\left(f_{1}(x), f_{2}(x), \ldots, f_{N}(x)\right)^{\mathrm{T}}
$$




$$
g_{j}(x)<0, j=1, \ldots M ; h_{h}(x)=0, k=1, \ldots K ; a(l) \leq x(l) \leq b(l), 1 \leq l \leq n
$$

Each of the $\mathrm{N}$ objective functions $f_{1}(x), f_{2}(x), \ldots, f_{N}(x)$ represents an objective that needs to be maximized or minimized throughout the optimization process. The terms $g_{j}(x)$ and $h_{h}(x)$ are called inequality and equality constraint functions, where $M$ and $K$ are the number of inequality and equality constraints. Besides, each $x(l)$ represents a gene (a variable) whose boundaries are defined by $a(l)$ and $b(l)$.Because $f(x)$ is a vector, if any of its components are competing, there is no unique solution to this problem. Instead, the concept of noninferiority (also called Pareto optimality) must be used to characterize the objectives [9].A Pareto optimal solution (nondominated by any other solution), may be mapped to different objective vectors, allowing for different trade-offs between objectives [9].

\section{LNA DESIGN USING COMPUTATIONAL INTELLIGENCE TECHNIQUES}

Multi-objective optimization using GA is now a commonly met technique in modern LNA design ([10] - [13]). Table 1 shows a chronological, comparative survey of the computational intelligence techniques involved, and also some specific values for the designed circuit.

Table 1. Types of computational intelligence techniques used in LNA design

\begin{tabular}{|l|l|l|l|}
\hline No. & \multicolumn{1}{|c|}{ Ref. } & \multicolumn{1}{|c|}{ Type } & \multicolumn{1}{c|}{ Observations } \\
\hline 1 & {$[10]-[13]$} & GA & - \\
\hline 2 & {$[14]$} & NSGA & ENSGA \\
\hline 3 & {$[15],[16]$} & NSGA & NSGA-II \\
\hline 4 & {$[17]$} & NSGA-II, MOO & Optimal bias conditions \\
\hline 5 & {$[18]$} & MOO & Adaptive normalized cost function \\
\hline 6 & {$[19]$} & GA & IMN/OMNdesign \\
\hline 7 & {$[20]$} & IGA & Layered encoding structure \\
\hline 8 & {$[21]$} & GA + expert system & \\
\hline 9 & {$[22]$} & GA + fuzzy logic & \\
\hline 10 & {$[23],[24]$} & GA + morphological & Pattern and morphological tree \\
\hline 11 & {$[25]$} & GA + SA & \\
\hline 12 & {$[26],[27]$} & GA + geometry & Geometry of the circuits' elements \\
\hline 13 & {$[28]$} & Honey-bee matching algorithm & FDTS \\
\hline
\end{tabular}

An elitist nondominated sorting genetic algorithm (ENSGA) is used in [14] to fully design an5.2 GHz direct-conversion receiver front-end, which includes a common-gate differential LNA, I/Q downconversionmixers, and a quadrature voltage-controlled oscillator, in 250-nm SiGeBiCMOStechnology.ENSGA is similar to other evolutionary techniques, in the sense that it uses multiple points in the solution space. The fundamental difference is that the solutions are distributed along the Pareto front, by means of the nondomination sorting mechanism with elitism. Unlike classical GA, the number of solutions is proportional to the number of objectives. The flowchart of the optimization is presented in Fig. 2. PVT analysis is used postoptimization.

NSGA-II is also used in [15], [16] to optimize the values of an LNA's components. Simulations were carried out via HSPICE RF. Two figures of merit evaluate the performance of the current design (7),(8):

$$
\begin{aligned}
& F O M_{1}=\frac{G[d B]}{N F[d B]} \\
& F O M_{2}=\frac{f[G H z] * G[a b s]}{N F[a b s] * V_{\text {supply }}[V]}(8)
\end{aligned}
$$

The algorithm reaches conversion after 600 generations, with a population of 100 individuals.

[21] uses an expert system to guide the optimization of RF circuits topologies. The knowledge base of the expert system consists of "If-Then"rules. A genetic algorithm using a custom cost function makes sure that a rescaling between 0 and 1 is performed, where more important factors have higher values. The LNA designed with this approach is noise figure optimized: the expert system picks the first available schematic, sees if it meet all the constraints, and if it does not, it will decide to sacrifice the NF in order to obtain a higher IIP3, for instance.

[19] proposes a GA based method for the design of the input and output matching networks (IMN/OMN) of an LNA. The chromosome consists of values for the components of IMN and OMN and a code for the topology. The fitness function is described as a weighted sum of the performance regarding the input and output match, and, respectively, of the gain and noise figure.

A multiobjective optimization method with adaptive normalized cost functions is applied in [18] to the design of an LNA. The optimization process is divided into two steps: objective function normalization and dynamic weights adjustment in the composite cost function. The nonlinear, parameterized normalization leads to better results than linear normalization methods. 
Interactive evolutionary computation is an evolutionary algorithm that includes subjective human evaluation. An IGA (interactive GA) is applied in [20] to optimize the design variables for a monolithic microwave integrated circuit LNA. A layered encoding structure (Fig. 3)is used as means of solution representation. In this case, layers represent different resolutions of the design variables, in order to narrow down the search space. Layers communicate between them, thus both the local and global optima are constantly updated.

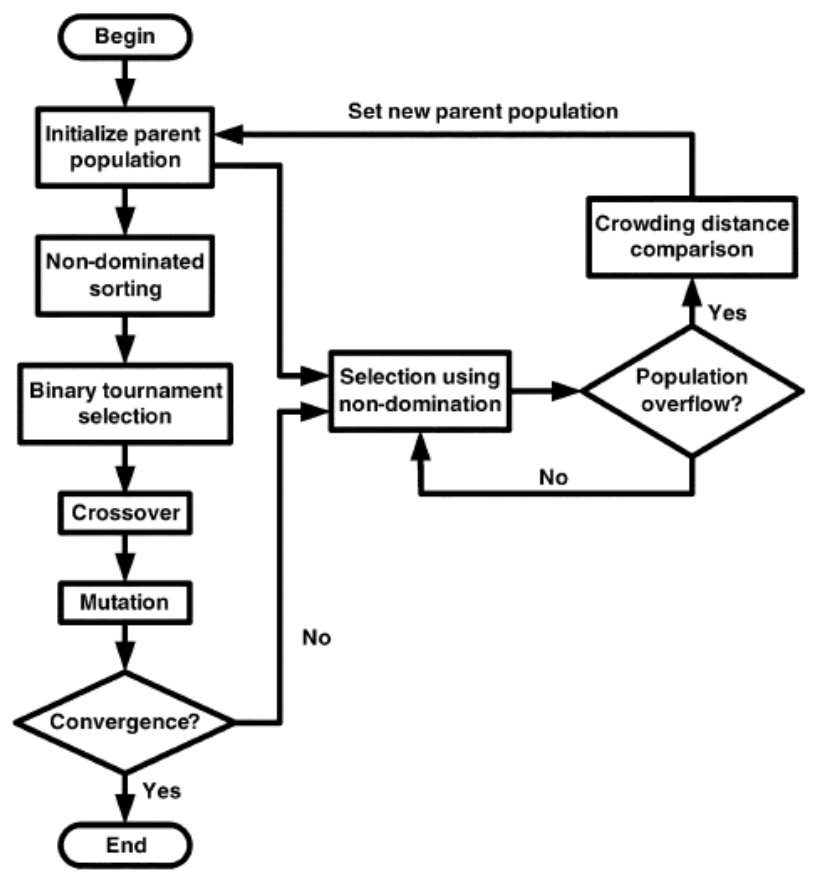

Figure 2.ENSGA flowchart [14]

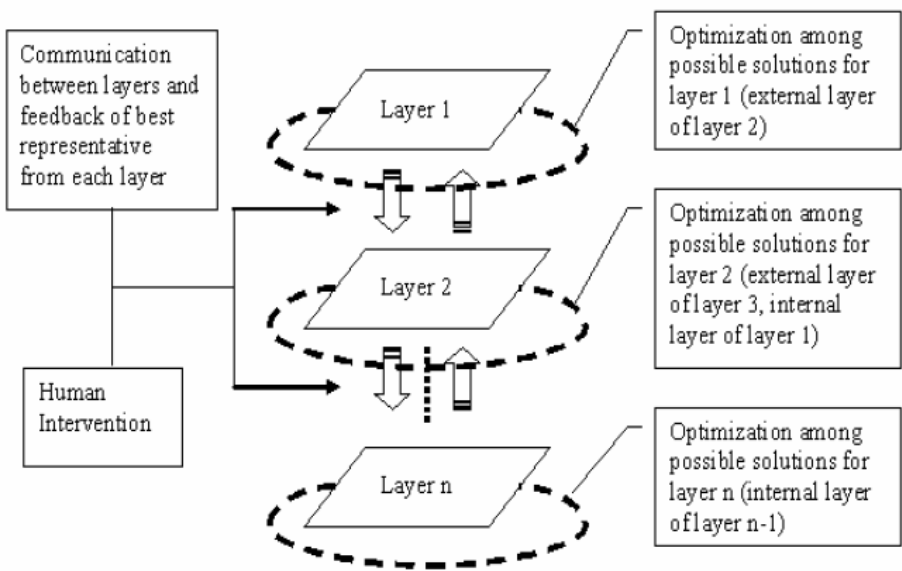

Figure 3. The layered encoding structure [20]

A combined method, using fuzzy logic as a human evaluator, and a GA, is presented in [22] (Fig. 4).Fuzzy decisions integrated in the GA optimization scheme increase the possible solutions base, by attempting to meet criteria for adequacy, rather than to find an optimal solution.

A GA combined with a morphological approach is described in [23] and [24]. The LNA structure is represented in the form of pattern and morphological tree. The method does not require a highly-skilled designer and it results in many possible variants of the circuit, from which the user can select. IDCSLNA.

Simulated annealing is combined with GA in [25] to optimize the electrical specifications of an 
The circuit elements' geometry is also part of the optimization process in [26], [27] and suggests an increase in speed, when compared to optimizing the components' values.

Optimal bias conditions for each stage of an LNA can also be obtained using GA techniques, namely binary GA and NSGA-II, as shown in [17].

The feasible design target space (FDTS) for a wideband front-end amplifier is successfully determined in [28], using a meta-heuristic optimization method, namely the honey bees mating algorithm.

\section{CONCLUSIONS}

Manual design of integrated circuits has lately become obsolete, given the fact that time constraints are more and more present. Hence, designers have shifted towards automatic approaches, using various software tools, which not only ensure a speedy result, but also provide a fertile environment for further designs. The paper presents a comparative study on how these techniques are used in low noise amplifiers (LNA) design. The LNA is a crucial block in the structure of a communications receiver and must meet some strict specifications. The survey shows that most commonly, some sort of multi-objective optimization is employed in the design process. Various combinations between genetic algorithms and other techniques, such as expert systems, fuzzy logic or simulated annealing are also present. Associating GAs with a morphological approach or searching for the optimum circuit elements' geometry, rather than for electrical values, are other modern ways to design an LNA.

The literature survey showed that although neural networks are a well-known and highly promising computational intelligence technique, they do not appear to be used for LNA design. This highlights the opportunity to investigate such an option, for instance to replace the circuit simulator in the optimization loop with a neural network model of the circuit, and to call on the simulator only for the final solution(s). Such an approach would certainly lead to a shorter overall design time and a reduced complexity.

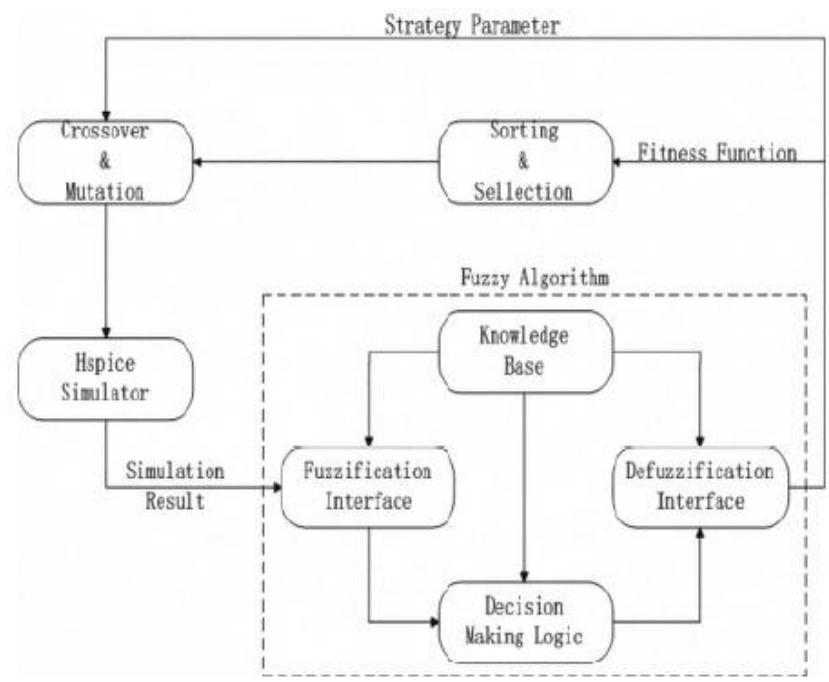

Figure 4. Basic configuration of Fuzzy Multi-objective Genetic Algorithm [22]

\section{Acknowledgements}

This paper was supported by the project "Improvement of the doctoral studies quality in engineering science for development of the knowledge based society-QDOC" contract no. POSDRU/107/1.5/S/78534, project co-funded by the European Social Fund through the Sectorial Operational Program Human Resources 2007-2013.

\section{REFERENCES}

[1] B. Razavi, RF Microelectronics, $2^{\text {nd }}$ Edition (Englewood Cliffs, NJ: Prentice-Hall, 2011).

[2] T.H. Lee, The Design of CMOS Radio-Frequency Integrated Circuits, Second Edition (Cambridge, UK: Cambridge University Press, 2003).

[3] D. Whitley,An Overview of Evolutionary Algorithms: Practical Issues and Common Pitfalls, Journal of Information and Software Technology, 43, 2001, 817-831

[4] R. Eberhart and Y. Shi, Computational Intelligence. Concepts to Implementations (Elsevier, Morgan Kaufman Publisher, 2007).

[5] D. Dumitrescu, Algoritmigeneticişistrategiievolutive - aplicaţiiînInteligenţaArtificialăşî̂ndomeniiconexe (Cluj-Napoca, Romania: EdituraAlbastră, 2006).

[6] T. Kalganova, Evolvable Hardware Design of Combinational Logic Circuits, doctoral diss., Napier University, Edinburgh, Scotland, 2000. 
[7] J.K. Li and H.C. Tseng, FOMs Extraction for Quantum-Well Lasers and Low-Noise Amplifiers With a Modified Genetic Algorithm Technique, Proc. 5th International Microsystems Packaging Assembly and Circuits Technology Conference, Taipei, Taiwan, 2010, $1-2$ [2010 fomfoms]

[8] G. Nicosia, S. Rinaudo, and E. Sciacca, An Evolutionary Algorithm-based Approach to Robust Analog Circuit Design using Constrained Multi-objective Optimization, Knowledge-based Systems,21(3), 2008, 175-183

[9] G. Oltean, S. Hintea, and E. Sipos: Analog Circuit Design Based on Computational Intelligence Techniques, Journal of Automation, Mobile Robotics \& Intelligent Systems, 3(2), 2009, 63-69

[10] M. Forouzanfar and S. Naseh, High Gain CMOSUWBLNA Employing Thermal Noise Cancellation, Proc. IEEE International Conference on Ultra-Wideband, Vancouver, Canada, 2009, 118-122

[11] E.C. Becerra-Alvarez, J.M. de la Rosa, and F. Sandoval, Design of a 1-V 90-nm CMOS Folded CascodeLNA for Multi-Standard Applications, Proc.53rd IEEE International Midwest Symposium on Circuits and Systems, Seattle, WA, 2010, 185-188

[12] L.W. Jer, G.L.J. Xiong, S. Chin, and A. Marzuki, GA-based Optimzation for Circuit Design Assistance, Proc. 3rd International Conference on Intelligent Systems, Modelling and Simulation, Kota Kinablu, Malaysia, 2012, 732-736

[13] S. Matindoust, G. SadeghyBajestani, I.A. Akhlaghi, H. Nabovati, and M. Vahedian, Improvement of Ultra-Wideband LNA Parameters; Multi-Objective Algorithm for Flatness Over Bandwidth Less 3dB, Proc. $20^{\text {th }}$ Iranian Conference on Electrical Engineering, Tehran, Iran, 2012, 154-159

[14] M. Chu and D.J. Allstot, Elitist Nondominated Sorting Genetic Algorithm Based RF IC Optimizer, IEEE Transactions on Circuits and Systems, 52(3), 2005, 535-545

[15] M.B. Fallahpour, K.D. Hemmati, A. Pourmohammad, and A. Golmakani, Multi Objective Optimization of a LNA Using Genetic Algorithm Based on NSGA-II, Proc. International Conference on Electrical Engineering and Informatics, Bandung, Indonesia, 2011, 1-4 [2011-moo lna nsga2]

[16] M.B. Fallahpour, K.D. Hemmati, and A. Pourmohammad, Optimization of a LNA Using Genetic Algorithm, Electrical and Electronic Engineering, 2(2), 2012, 38-42

[17] A. Prasad, M. Roy, A. Biswas, and D. George, Application of Genetic Algorithm to Multi-objective Optimization in LNA Design, Proc. Asia-Pacific Microwave Conference, 2010, 362-365

[18] A. Somani, P.P. Chakrabarti, and A. Patra, An Evolutionary Algorithm-Based Approach to Automated Design of Analog and RF Circuits Using Adaptive Normalized Cost Functions, IEEE Transactions on Evolutionary Computation, 11(3), 2007, 336-353

[19] H.H. Chen, M.H. Chen, and C.Y. Tsai, Optimization of Low Noise Amplifier Designs by Genetic Algorithms, Proc. International Symposium on Electromagnetic Theory, Hiroshima, Japan, 2013, 493-497

[20] N. Siew Chin, M. Arjuna, M. Norhashimah, L. CheePeng, and A.A. Zalina, An Interactive Genetic Algorithm Approach to MMIC Low Noise Amplifier Design Using A Layered Encoding Structure, Proc. IEEE Congress on Evolutionary Computation, Hong Kong, China, 2008, 1571-1575

[21] G. Konstantopoulos, K Papathanasiou, and A. Samelis, Optimization of RF Circuits by Expert System monitored Genetic Computation, Proc. IEEE International Symposium on Circuits and Systems, Kos, Greece, 2006, 5247-5250

[22] X. Wang, C. Zhou, Z. Zhang, T. Ren, and L. Liu, Optimal RF IC design Based on Fuzzy Genetic Algorithm, Proc. PrimeAsia, Shanghai, China, 2009, 229-232

[23] L.I. Babak, A.A. Kokolov, and A.A. Kalentyev, A New Technique for Syntesis of Low Noise Amplifiers Based on Genetic Algorithm and Morphological Approach, Proc. $21^{\text {st }}$ International Crimean Conference "Microwave and Telecommunication Technology", Sevastopol, Ukraine, 2011, 228-229

[24] L.I. Babak, A.A. Kokolov, A.A. Kalentyev, and D.V. Garays, A New Genetic-Algorithm-Based Technique for Low Noise Amplifier Synthesis, Proc. $7^{\text {th }}$ European Microwave Integrated Circuits Conference, Amsterdam, The Netherlands, 2012, 381-384

[25] S. Ehrampoosh, A. Hakimi, and H.R. Naji, An Optimized High Gain CMOSLNA using Simulated Annealing and Modified Genetic Algorithm, Proc. $19^{\text {th }}$ Iranian Conference on Electrical Engineering, Tehran, Iran, 2011, 1-6

[26] F. Gunes, A.K. Keskin, and S. Demirel, Genetic Algorithm Applied to Microstrip Implementation of Matching Circuits for a UWB Low-Noise Amplifier, Proc. IEEE International Conference on Ultra-Wideband, Syracuse, NY, 2012, 241-245

[27] A. Fathianpour, and S. Seyedtabaii, On the Intelligent Components Geometry Design of a Current Reuse Folded Cascode Concurrent Dual-band CMOSLNA, Proc. $21^{\text {th }}$ Iranian Conference on Electrical Engineering, Mashaad, Iran, 2013, 1-6

[28] P. Mahouti, F. Gunes, and S. Demirel, Honey-Bees Mating Algorithm Applied to Feasible Design Target Space for a Wide-Band Front-End Amplifier, Proc. IEEE International Conference on Ultra-Wideband, Syracuse, NY, 2012, 251-255 